\title{
Resting metabolic rate in older adults with overweight and obesity: differences in measured versus predicted values
}

\author{
C. Cooney ${ }^{1}$ and L. Ryan ${ }^{1}$ \\ ${ }^{1}$ Department of Sport, Exercise and Nutrition, School of Science and Computing, Galway-Mayo Institute of \\ Technology Galway Campus, Galway, Ireland.
}

A low relative resting metabolic rate (RMR) is predictive of weight gain over time thus an accurate assessment of RMR is fundamental to the determination of nutritional requirements in adult weight management. The objective of the present study was to compare measured RMR with values for RMR calculated from prediction equations in older adults with overweight and obesity.

Fifty-two adults aged 50 years or greater with a BMI greater than or equal to $25 \mathrm{~kg} / \mathrm{m} 2$ were recruited to participate in this study. Participants were excluded from the study if they had any condition or were taking medication known to influence the measurement of RMR or body composition. RMR was measured by indirect calorimetry after an overnight fast in 28 female (age $55.3 \pm 5.1$ years, BMI $30.5 \pm 4.7 \mathrm{~kg} / \mathrm{m} 2$ ) and 24 male (age $58.2 \pm 6.1$ years, BMI $31.0 \pm 4.6 \mathrm{~kg} / \mathrm{m} 2$ ) participants. Measured values were compared with predicted values determined by Harris and Benedict ${ }^{(1)}$, Bernstein et al. ${ }^{(2)}$, FAO/WHO/UNU (Food and Agricultural Organisation/World Health Organisation/United Nations University) ${ }^{(3)}$, Owen et al. ${ }^{(4,5)}$, Mifflin et al. ${ }^{(6)}$, Weijs and Vansant ${ }^{(7)}$ predic- $^{\circ}$ tion equations.

The Bernstein ${ }^{(2)}$, Owen (FFM) $)^{(4)}$ and Mifflin et al. (FFM) ${ }^{(6)}$ prediction equations significantly underestimated RMR in females and the Weijs and Vansant ${ }^{(7)}$ significantly overestimated RMR when compared to measured RMR (1467 $\pm 347 \mathrm{kcal} /$ day). The Bernstein (weight and FFM) ${ }^{(2)}$, FAO/WHO/UNU (>60 years) $)^{(3)}$, Owen (weight and FFM) ${ }^{(5)}$ Mifflin et al. (weight and FFM) ${ }^{(6)}$ prediction equations significantly underestimated RMR in males when compared to measured RMR (2061 $\pm 411 \mathrm{kcal} / \mathrm{day})$.

The Harris and Benedict ${ }^{(1)}$, Mifflin et al. ${ }^{(6)}$ using actual weight, and FAO/WHO/UNU(3)for 30-60 and $>60$ years are the most appropriate prediction equations for estimating RMR in female adults aged 50 years and older with overweight or obesity. The Weijs and Vansant ${ }^{(7)}, \mathrm{FAO} / \mathrm{WHO} / \mathrm{UNU}^{(3)}, 30-60$ years and Harris and Benedict ${ }^{(1)}$ are the most appropriate prediction equations for estimating RMR in male adults aged 50 years and older with overweight or obesity. For an estimate of RMR in all adults both male and female, the results of this study suggest the Harris and Benedict ${ }^{(1)}$ and FAO/WHO/UNU ${ }^{(3)} 30-60$ years provide accurate prediction. However, these are estimates and caution should be employed when applying prediction equations to specific populations along with clinical judgment when adjusting nutrition plans to avoid excessive energy restriction or overfeeding. Overall, having better estimations of RMR, particularly for older adults with overweight and obesity, may help existing public health efforts addressing overweight and obesity that rely on lifestyle interventions based, in part, on energy requirements.

\section{Acknowledgements}

The authors are grateful to all the participants for their time in contributing to this research.

\section{References}

1. Harris JA \& FG Benedict (1918) PNAS 4(12), 370-373.

2. Bernstein RS, et al. (1983) Am J Clin Nutr 37(4), 595-602.

3. FAO/WHO/UNU (1985), Energy and protein requirements. Report of a joint FAO/WHO/UNU Expert Consultation. World Health Organisation Technical Report Series, 724, 1-206.

4. Owen OE, et al. (1986) Am J Clin Nutr 44(1), 1-19.

5. Owen OE, et al. (1987) Am J Clin Nutr 46(6), 875-85.

6. Mifflin MD, et al. (1990) Am J Clin Nutr 51(2), 241-7.

7. Weijs PJM \& Vansant GAA (2010) Clin Nutr 29(3), 347-351. 